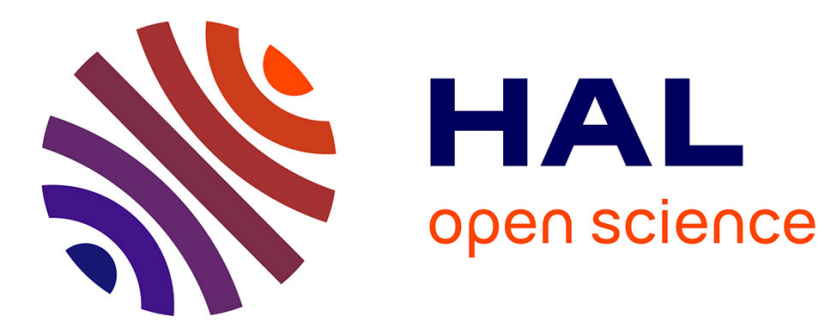

\title{
Radio Resource Sharing for MTC in LTE-A: An Interference-Aware Bipartite Graph Approach
}

\author{
Safa Hamdoun, Abderrezak Rachedi, Yacine Ghamri-Doudane
}

\section{To cite this version:}

Safa Hamdoun, Abderrezak Rachedi, Yacine Ghamri-Doudane. Radio Resource Sharing for MTC in LTE-A: An Interference-Aware Bipartite Graph Approach. IEEE Global Communication Conference (IEEE Globecom), Dec 2015, San Diego, CA, United States. hal-01171611

\section{HAL Id: hal-01171611 \\ https://hal.science/hal-01171611}

Submitted on 14 Oct 2015

HAL is a multi-disciplinary open access archive for the deposit and dissemination of scientific research documents, whether they are published or not. The documents may come from teaching and research institutions in France or abroad, or from public or private research centers.
L'archive ouverte pluridisciplinaire HAL, est destinée au dépôt et à la diffusion de documents scientifiques de niveau recherche, publiés ou non, émanant des établissements d'enseignement et de recherche français ou étrangers, des laboratoires publics ou privés. 


\section{Radio Resource Sharing for MTC in LTE-A: An Interference-Aware Bipartite Graph Approach}

\author{
Safa Hamdoun \\ Université Paris-Est \\ LIGM (UMR8049, UPEM) \\ Email: hamdoun@u-pem.fr
}

\author{
Abderrezak Rachedi \\ Université Paris-Est \\ LIGM (UMR8049, UPEM) \\ Email: rachedi@u-pem.fr
}

\author{
Yacine Ghamri-Doudane \\ L3i Lab, Pôle Sciences et Technologie \\ Université de La Rochelle \\ Email: yacine.ghamri@univ-lr.fr
}

\begin{abstract}
Traditional cellular networks have been considered the most promising candidates to support machine to machine (M2M) communication mainly due to their ubiquitous coverage. Optimally designed to support human to human $(\mathrm{H} 2 \mathrm{H})$ communication, an innovative access to radio resources is required to accommodate M2M unique features such as the massive number of machine type devices (MTDs) as well as the limited data transmission session. In this paper, we consider a simultaneous access to the spectrum in an $\mathrm{M} 2 \mathrm{M} / \mathrm{H} 2 \mathrm{H}$ coexistence scenario. Taking the advantage of the new device to device (D2D) communication paradigm, enabled in long term evolution-advanced (LTE-A), we propose to combine M2M and D2D owing to the MTD low transmit power and thus enabling efficiently resource sharing. First, we formulate the resource sharing problem as a maximization of the sum-rate, problem for which the optimal solution has been proved to be non deterministic polynomial time hard (NP-Hard). We next model the problem as a novel interference-aware bipartite graph to overcome the computational complexity of the optimal solution. To solve this problem, we consider here a two-phase resource allocation approach. In the first phase, H2H users resource assignment is performed in a conventional way. In the second phase, we introduce two alternative algorithms, one centralized and one semi-distributed to perform the M2M resource allocation. The computational complexity of both introduced algorithms whose aim is to solve the M2M resource allocation, is of polynomial complexity. Simulation results show that the semi-distributed M2M resource allocation algorithm achieves quite good performance in terms of network aggregate sum-rate with markedly lower communication overhead compared to the centralized one.
\end{abstract}

Index Terms-Machine to machine (M2M), human to human $(\mathrm{H} 2 \mathrm{H})$, device to device (D2D), bipartite graph (BP), resource sharing.

\section{INTRODUCTION}

Machine to machine (M2M) communication is a new paradigm that enables the ubiquitous connectivity between a myriad of machines without or with limited human intervention. Thus, the autonomous connection of devices facilitates the emergence of a wide range of intelligent M2M applications. These latter have exhibited a strong potential to improve human life in different fields such as eHealth, smart grids, smart home, intelligent transportation and surveillance, enabling partially the internet of things (IoT) [1], [2].

Cellular wireless technologies have been considered the most promising candidates to support $\mathrm{M} 2 \mathrm{M}$ communication for its ubiquitous coverage, good support of user mobility, high data rates and flexible spectrum usage. Consequently, the third generation partnership project (3GPP) has standardized M2M as machine type communication (MTC) in long term evolution and its advancements (LTE-A). 3GPP has been investigating in release 10 and beyond potential problems posed by MTC on their cellular networks optimally designed for human to human $(\mathrm{H} 2 \mathrm{H})$ communication [3], [4]. For instance, a cellular MTC reference architecture was specified by 3GPP. Besides, an effort on enabling low cost machine type devices (MTDs) is considered [5].

Unlike traditional $\mathrm{H} 2 \mathrm{H}$ applications, M2M services have their own specific features: time-tolerant, small data transmission, extra low power consumption and centralized data collection that render M2M uplink scheduling a critical issue to solve. Traditional LTE schedulers have been designed to carry high data rates for broadband applications where the majority of schedulers exploit channel quality on a fast time scale (per transmission time interval (TTI) ideally) [6]. Consequently, the associated signaling load and complexity render existing LTE uplink schedulers prohibitive to cater to M2M requirements.

Along with the MTC new paradigm, 3GPP has introduced a new technology called device to device (D2D) communication for LTE-A in release 12. D2D is defined as the direct communication between two users without traversing the base station [7]. Both D2D and cellular user equipments (CUEs) links share the same radio resources. Thus, new resource allocation methods should be developed to mitigate the cochannel interference.

In this paper, we propose the design of efficient and dynamic resource allocation schemes to support MTC without significantly degrading the performance of $\mathrm{H} 2 \mathrm{H}$ services. Due to the low MTD transmission power, we propose to aggregate D2D and MTC concepts in order to increase the density of connections per cell. More precisely, we consider here the occurrence of simultaneous access to radio resources between CUEs and MTD within D2D communication. Hence, we formulate the resource sharing problem as an interferenceaware bipartite graph. We consider here a two-phase resource allocation approach. In the first phase, higher priority is given to CUEs where conventional schedulers are used. In the second phase, we propose two alternative MTC radio resource sharing algorithms: a centralized one and a semi-distributed one. Finally, we evaluate the network performance as well 
as the impact of the introduction of MTC on existing $\mathrm{H} 2 \mathrm{H}$ services.

The remainder of this paper is organized as follows. In Section II, we present some already existing approaches for M2M scheduling. The specific technical details of the scenario under evaluation is described and the corresponding resource sharing problem is formulated in Section III. In Section IV, an interference-aware bipartite graph based resource sharing scheme is proposed. The performance evaluation of our proposal is drawn in section $\mathrm{V}$ followed by the conclusion in Section VI.

\section{RELATED WORK}

Since most MTC applications are uplink-centric, literature addressing uplink scheduling for M2M in LTE-A has gained much momentum even though it is still scarce due to the recent appearance of this new paradigm. Authors in [8] have proposed a random access method for MTDs in LTE-A. However, significant congestions may occur when there is a high number of MTDs. A QoS guaranteed M2M massive access management scheme has been proposed in [9], [10]. In this scheme, MTDs sharing the same QoS requirements, i.e. the jitter, are grouped logically into clusters. Then, fixed access grant time interval (AGTI) is allocated to each cluster. The major limitation is that contrarily to the randomness of real M2M traffic, only constant-rate traffic patterns have been considered by the authors. Furthermore, the impact of this mechanism on CUEs has not been discussed.

In [11], authors have proposed two fully dynamic (per transmission time interval, TTI) M2M scheduling algorithms for the LTE uplink based on a delay tolerance objective and channel conditions. Higher priority is given to CUEs, then the remaining resources are assigned to MTDs. In [12], a mixed scheduler for $\mathrm{H} 2 \mathrm{H}$ and $\mathrm{M} 2 \mathrm{M}$ communications has been introduced. Authors have differentiated between $\mathrm{H} 2 \mathrm{H}$ and M2M services and have provided schedulers for each of them giving higher priority to CUEs. The major drawbacks of these algorithms are the starvation of MTDs in case of heavy $\mathrm{H} 2 \mathrm{H}$ traffic and the associated signaling load due to the use of a centralized approach where resource allocation is performed individually.

It is important to notice that all the proposed methods have used a centralized approach that generate high complexity and lead to a huge signaling load. In addition, all these methods have considered orthogonal scheduling. Indeed, a certain amount of resources is reserved for CUEs while the residual part is assigned to MTDs. Finally, the impact on CUEs communication has not been discussed.

In this paper, we first propose to aggregate in-range MTDs and D2D to support a higher density of connections per cell and cater to the scalability issue. Then, we formulate the radio resource sharing process between coexisting CUEs and MTDs as an interference-aware bipartite graph. To overcome the above mentioned limitations of the existing schemes, we adopt a two phase M2M resource allocation approach. In the first phase, conventional schedulers optimally designed for

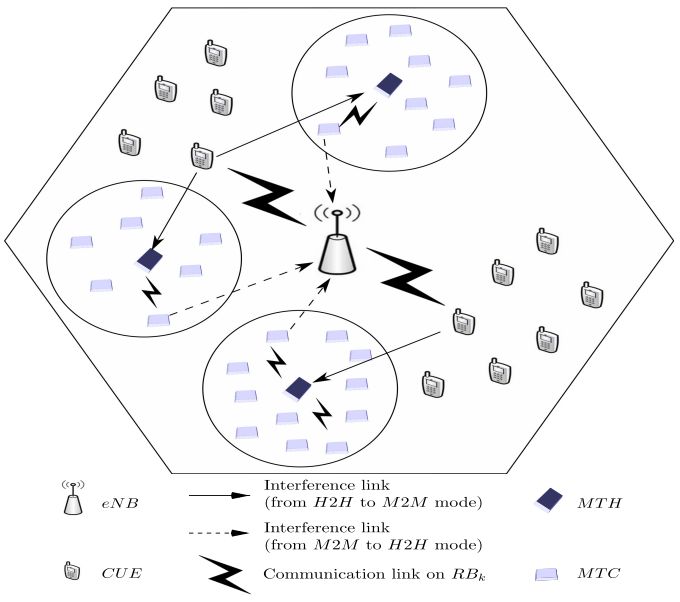

Fig. 1: Scenario model under evaluation: MTC within D2D underlaying cellular network when sharing uplink resources

$\mathrm{H} 2 \mathrm{H}$ communication are used for CUEs. In the second phase, we propose two alternative M2M schedulers (one centralized and one semi-distributed) based on the resource assignment solution given in the first phase. To the best of the authors's knowledge, our work is pioneer in dealing with radio resource sharing for physically group-based MTDs within D2D communication in a joint $\mathrm{M} 2 \mathrm{M} / \mathrm{H} 2 \mathrm{H}$ coexistence scenario.

\section{System Model and Problem Formulation}

\section{A. System Description}

As illustrated in Fig 1, we consider an uplink single cell scenario with $N$ traditional CUEs, $M$ MTDs per cluster and one evolved NodeB (eNB) located at the center of the cell. We suppose that $L$ is the number of clusters. Thus, there are $N+L \times M$ uplink flows in the considered cell. MTDs communicate through D2D communication, making MTC as an underlay to $\mathrm{H} 2 \mathrm{H}$ communication. For simplicity, we assume that D2D corresponds to local uplink communication between MTDs and a machine type cluster head (MTH). We consider the use of the single carrier frequency division multiple access (SC-FDMA) for LTE uplink transmission that requires resource block $(\mathrm{RB})$ contiguity constraint. Note that a $\mathrm{RB}$ is the minimum unit of the resource allocation process with a bandwidth of $180 \mathrm{Khz}$, that consists of 12 subcarriers of 15 Khz each [13]. We assume that CUEs are not allowed to share the same RB due to their relatively high transmission power, implying that there is no interference from CUEs to eNB. Moreover, we suppose that MTDs are allocated at most one RB due to their small data transmission. No MTDs belonging to the same cluster share the same RB, implying that there will be no intra-cluster interference. Resource sharing among CUEs and MTDs as well as among MTDs of different clusters is allowed owing to the MTD low transmit power. We focus on the intra-cell interference, particularly the interference from CUEs to MTHs (interference from $\mathrm{H} 2 \mathrm{H}$ to $\mathrm{M} 2 \mathrm{M}$ mode) and from MTDs to the eNB (interference from M2M to $\mathrm{H} 2 \mathrm{H}$ mode). 
Let's define the following sets of elements:

$$
\begin{array}{ll}
\mathcal{K}=\{1,2, \cdots, K\} & \text { set of subchannels; } \\
\mathcal{M}=\{1,2, \cdots, M\} & \text { set of MTDs; } \\
\mathcal{N}=\{1,2, \cdots, N\} & \text { set of CUEs; } \\
\mathcal{L}=\{1,2, \cdots, L\} & \text { set of clusters; } \\
P_{n} & \text { CUE transmit power; } \\
P_{m} & \text { MTD transmit power; } \\
U_{n} & \text { Traditional CUE; } \\
C_{l} & \text { Cluster } l ; \\
D_{m, t}^{l} & \text { MTD transmitter m that belongs to } C_{l} ; \\
D_{r}^{l} ; & \text { MTH or MTD receiver of } C_{l} .
\end{array}
$$

Similarly to [14], the channel gains of the different communication and interference links, determined from the path loss (PL) and the small scale fading, are listed in Table I. We assume a frequency flat fading in each RB where the small scale fading for a certain communication link is independent, but remains the same on each RB.

TABLE I: CHANNEL GAINS

\begin{tabular}{|c|c|}
\hline Channel gain on $R B_{k}$ & from $\Rightarrow$ to \\
\hline$g_{U_{n}}^{k}=P L_{U_{n}} h_{U_{n}}^{k}$ & $\mathrm{UE} \Rightarrow \mathrm{eNB}$ \\
\hline$g_{D_{m, t}^{l}, D_{r}^{l}}^{k}=P L_{D_{m, t}^{l}, D_{r}^{l}} h_{D_{m, t}^{k}, D_{r}^{l}}^{k}$ & $\mathrm{MTD} \Rightarrow \mathrm{MTH}$ \\
\hline$g_{D_{m, t}^{k}, e N B}^{k}=P L_{D_{m, t}^{l}, e N B} h_{D_{m, t}^{k}, e N B}^{l}$ & $\mathrm{MTD} \Rightarrow \mathrm{eNB}$ \\
\hline$g_{U_{n}, D_{r}^{l}}^{k}=P L_{U_{n}, D_{r}^{l}} h_{U_{n}, D_{r}^{l}}^{k}$ & $\mathrm{UE} \Rightarrow \mathrm{MTH}$ \\
\hline
\end{tabular}

We evaluate the instantaneous Signal-to-Interference-plusNoise-Ratio (SINR) at the CUE $U_{n}, n \in \mathcal{N}$ when the resource block, $R B_{k}$, is assigned to it as:

$$
S I N R_{U_{n}}^{k}=\frac{P_{n} g_{U_{n}}^{k}}{\sum_{l \in L} \sum_{j \in M} P_{m} g_{D_{j, t}^{l}, e N B}^{k}+\sigma^{2}}
$$

where the first term in the denominator represents the interference from the MTDs of different clusters, sharing the $R B_{k}$ with the CUE, to the eNB and the second term represents the variance of the thermal noise, denoted by $\sigma^{2}$ and modeled as an independent Gaussian distribution with zero mean.

We evaluate the instantaneous SINR at each MTH $D_{r}^{l}, l \in \mathcal{L}$ when $R B_{k}$ is assigned to it as:

$$
S I N R_{D_{r}^{l}}^{k}=\frac{P_{m} g_{D_{m, t}^{l}, D_{r}^{l}}^{k}}{\sum_{i \in N, U_{i}} P_{n} g_{U_{i}, D_{r}^{l}}^{k}+\sum_{l^{\prime} \in L, l \neq l^{\prime}} \sum_{j \in M} P_{m} g_{D_{j, t}^{l^{\prime}}, D_{r}^{l}}^{k}+\sigma^{2}}
$$

where the first term in the denominator represents the interference from CUEs to the MTH $\left(D_{r}^{l} \in C_{l}, l \in \mathcal{L}\right)$, while the second term represents the inter-cluster interference that consists of the interference on $R B_{k}$ caused by MTDs of different clusters $\left(D_{m, t}^{l^{\prime}} \in C_{l^{\prime}}, l \neq l^{\prime}\right)$ to the $\mathrm{MTH}\left(D_{r}^{l} \in C_{l}\right)$.

\section{B. Resource Sharing Problem}

After defining our system model, let us now investigate the resource allocation problem in the uplink transmission case for group-based MTDs within D2D underlaying CUEs. The goal is to maximize the sum of the Shannon capacity within the network involving both $\mathrm{H} 2 \mathrm{H}$ and MTC communication links. Let us define $\alpha_{n \times k}$ and $\beta_{m \times k}^{l}$ as the RB assignment matrices for both CUEs and in-range MTDs, respectively.

$$
\alpha_{n \times k}= \begin{cases}1, & \text { if } R B k \text { is allocated to } U_{n} \\ 0, & \text { otherwise. }\end{cases}
$$

and

$$
\beta_{m \times k}^{l}= \begin{cases}1, & \text { if } R B k \text { is allocated to } D_{m, t}^{l} \\ 0, & \text { otherwise. }\end{cases}
$$

Hence, we can obtain the optimal RB assignment solution by solving the following optimization problem:

$$
\begin{array}{r}
S_{\text {opt }}=\arg \max _{S_{(N+L \times M) \times K}} \sum_{k=1}^{K} \frac{W}{K}\left[\sum_{n=1}^{N} \log _{2}\left(1+S I N R_{U_{n}}^{k}\right) \alpha_{n \times k}\right. \\
\left.+\sum_{l=1}^{L} \sum_{m=1}^{M} \log _{2}\left(1+S I N R_{D_{r}^{l}}^{k}\right) \beta_{m \times k}^{l}\right]
\end{array}
$$

subject to

$$
\left\{\begin{aligned}
& \sum_{n=1}^{N} \alpha_{n \times k} \leq 1, \leq \\
& \sum_{m=1}^{M} \beta_{m \times k}^{l} \leq 1, \\
& \sum_{k=1}^{K} \beta_{m \times k}^{l} \leq 1
\end{aligned}\right.
$$

Where $W$ is the channel bandwidth and $S I N R_{U_{n}}^{k}$ and $S I N R_{D_{r}^{l}}^{k}$ are given respectively by (1) and (2). Note that the first constraint in (4) guarantees the exclusivity for occupying the same RB by traditional CUEs. The second constraint in (4) guarantees that two MTDs belonging to a same cluster are not allowed to re-use the same RB. The third constraint in (4) ensures that at most one RB is assigned to MTC links.

\section{Complexity analysis}

The optimal RB assignment $S_{o p t}$ solution in (3) can be obtained through an exhaustive search for all $\alpha_{n \times k}$ and $\beta_{m \times k}^{l}$ in each cluster subject to the constraints in (4). The computational complexity to allocate a set of RBs to $N \mathrm{H} 2 \mathrm{H}$ users considering all the possible choices while taking into account the first constraint in (4) is $\mathcal{O}\left(\frac{N !}{(N-K) ! K !}\right)$. Meanwhile the computational complexity when considering all of the possible choices of RB assignment to MTDs in each cluster subject to the second and third constraint in (4) can be computed in $\mathcal{O}\left(M^{L K}\right)$. Thus, the total computational complexity of the optimal RB assignment solution is given by:

$$
\mathcal{C}_{S_{\text {opt }}}=\mathcal{O}\left(\sum_{k=0}^{K} \frac{N !}{(N-k) ! k !} \times M^{L k}\right)
$$

Hence, the computational complexity obtained in (5) is exponential. From [15], [14], the optimization problem of (3) is a non deterministic polynomial time hard (NP-Hard) combinatorial optimization problem with non linear constraints.

\section{INTERFERENCE-AWARE BIPARTITE GRAPH RESOURCE SHARING ALGORITHMS}

Due to the inherent high complexity of solving the optimization problem of (3), we propose a bipartite graph (BG) based scheduling approach to provide a suboptimal solution for the resource sharing in a joint $\mathrm{M} 2 \mathrm{M} / \mathrm{H} 2 \mathrm{H}$ coexistence scenario. At the aim of not sacrificing $\mathrm{H} 2 \mathrm{H}$ services, we propose a two-stage resource allocation approach. In the first stage, traditional CUEs are given higher priority. Consequently, CUEs resource assignment is performed exclusively where 
conventional schedulers (proportional fair $(P F)$ scheduler, round robin $(R R)$ scheduler) [16] optimally designed for $\mathrm{H} 2 \mathrm{H}$ users are used. Note that there is no MTC links sharing any of the RBs in this phase. In the second stage, we propose the following two alternative MTC resource allocation algorithms.

\section{A. Bipartite Graph modeling}

First, let us represent the MTD resource sharing assignment problem as a BG. The goal is to re-assign RBs to MTC links, already allocated to CUEs in a way to minimize the total interference, and thus enhance the network throughput. A weighted BG $G=(U, E)$ is constructed, where the vertices are divided into two disjoint subsets, $U_{n, k}$ and $U_{m}$. While $U_{n, k}$ is the pair $(U E, R B)$ given by the sub-channel allocation solution, $\bar{\alpha}$, when applying traditional $\mathrm{H} 2 \mathrm{H}$ resource allocation algorithms, $U_{m}$ represents the set of MTDs, $\bigcup_{l=1}^{L} D_{m, t}^{l}$. Each vertex in $U_{n, k}$ is neighbor to all vertices in $U_{m}$. According to the proposed scenario in Fig 1, nodes in subset $U_{m}$ are divided into $L$ clusters, $U_{m}=\bigcup_{l=1}^{L} U_{m}^{l}$. It is clear that $U=U_{m} \cup U_{n, k}$ and $U_{m} \cap U_{n, k}=\varnothing$. Each edge, denoted by $e_{i, j}^{l}$, with one endpoint in $U_{n, k}$ and the other one in $U_{m}^{l}$ represents the RB assigned to a CUE and re-used by MTC links. The set of edges of $G$ are denoted by:

$$
E=\bigcup_{l=1}^{L} E^{l}=\bigcup_{l=1}^{L} e_{i, j}^{l} \mid u_{i} \in U_{n, k}, u_{j} \in U_{m}^{l}
$$

We express the weight assigned to edges by the potential mutual interference when re-using the same RB. Thus, we sum the interference from $\mathrm{H} 2 \mathrm{H}$ to $\mathrm{M} 2 \mathrm{M}$ and vice-versa on the RBs. In Fig 2 (a), an example of $G$ in a network with $U_{n, k}=4, U_{m}=5$ and $L=2$ is illustrated.

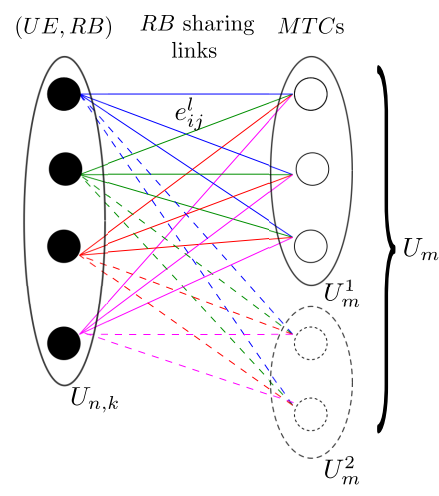

(a)

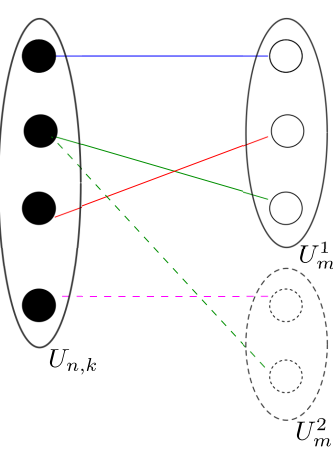

(b)
Fig. 2: Exemplary of a bipartite graph and its matching $U_{m}=5, U_{n, k}=4$

\section{B. Centralized MTC Resource Allocation approach (C-MTC-} $R A)$

Based on the knowledge of the path loss and fading for each interference link on each $R B_{k}$, the eNB calculates the accurate interference power that will be associated to the weight of each edge. We define the weight of each edge, $w\left(e_{i, j}^{l}\right)$, by the sum of the mutual interference power between two vertices expressed in (i) and (ii) as:

(i) Interference caused by $\mathrm{H} 2 \mathrm{H}$ mode on $\mathrm{M} 2 \mathrm{M}$ mode: Considering the M2M mode as the primary mode; it represents the interference, $I_{\left(U_{n} \rightarrow D_{r}^{l}\right)}$, caused by $U_{n}$ to $D_{r}^{l}$ on $R B_{k}$.

$$
I_{\left(U_{n} \rightarrow D_{r}^{l}\right)}^{k}=P_{n} g_{U_{n}, D_{r}^{l}}^{k}
$$

(ii) Interference caused by $M 2 \mathrm{M}$ mode on $\mathrm{H} 2 \mathrm{H}$ mode: Considering the $\mathrm{H} 2 \mathrm{H}$ mode as the primary mode; it represents the interference, $I_{\left(D_{m, t}^{l} \rightarrow e N B\right)}^{k}$, caused by $D_{m, t}^{l}$ to eNB on $R B_{k}$.

$$
I_{\left(D_{m, t}^{l} \rightarrow e N B\right)}^{k}=P_{m} g_{D_{m, t}^{l}, e N B}^{k}
$$

\section{- The weighted BG solution}

For each MTH $\left(D_{r}^{l}\right)$, we obtain a matching of $G$, denoted by $M^{l}$ (see Fig 2 (b)). $M^{l}$ consists of the subset of the edges in $G$ where each pair of edges in $U_{m}^{l}$ has no common ends. A match $M^{l}$ for each cluster is defined as follows:

- $M^{l} \subseteq E^{l}$

- If $e_{i, j}^{l} \in M^{l} \mid e_{i, y \neq j}^{l} \notin M^{l} \wedge e_{x \neq i, j}^{l} \notin M^{l}$

Here, the eNB acts as a scheduling operator that communicates the decisions on RB allocation to each MTH (see Algorithm 1). The minimum weighted matching (MWM) problem of the given BG $G$ satisfies that:

$$
W_{\text {opt }}=\min \sum_{e_{i, j}^{l} \in M^{l}} w\left(e_{i, j}^{l}\right), l \in \mathcal{L}
$$

To solve this problem, we use the Kuhn Munkres algorithm that has been proved to achieve MWM for BGs [17].

\section{Semi-Distributed MTC Resource Allocation approach ( $s D$ - MTC-RA)}

Compared to the centralized method based on the accurate knowledge of the channel gains, only the current locations of CUEs and MTDs as well as the solution $\bar{\alpha}$ that are not greedy in terms of resources are required. A path loss model is used to compute the interference power without the need of accurate interference channel information. The interference is calculated as the sum of the following interferences given by (i) and (ii) as:

(i) Interference caused by $\mathrm{H} 2 \mathrm{H}$ mode on $\mathrm{M} 2 \mathrm{M}$ mode: Considering the M2M mode as the primary mode; it represents the interference caused by the $U_{n}$ to the $D_{r}^{l}$.

$$
I_{\left(U_{n} \rightarrow D_{r}^{l}\right)}=c\left(d_{U_{n}-D_{r}^{l}}\right)^{-\alpha} P_{n}
$$

(ii) Interference caused by $M 2 \mathrm{M}$ mode on $\mathrm{H} 2 \mathrm{H}$ mode: Considering the $\mathrm{H} 2 \mathrm{H}$ mode as the primary mode; it represents the interference caused by $D_{m, t}^{l}$ to the eNB.

$$
I_{\left(D_{m, t}^{l} \rightarrow e N B\right)}=c\left(d_{D_{m, t}^{l}-e N B}\right)^{-\alpha} P_{m}
$$

Where $I_{(x \rightarrow y)}$ means the interference power from x to $\mathrm{y}, d_{x-y}$ denotes the distance between $\mathrm{x}$ and $\mathrm{y}$ nodes, $\mathrm{c}$ and $\alpha$ are a path 


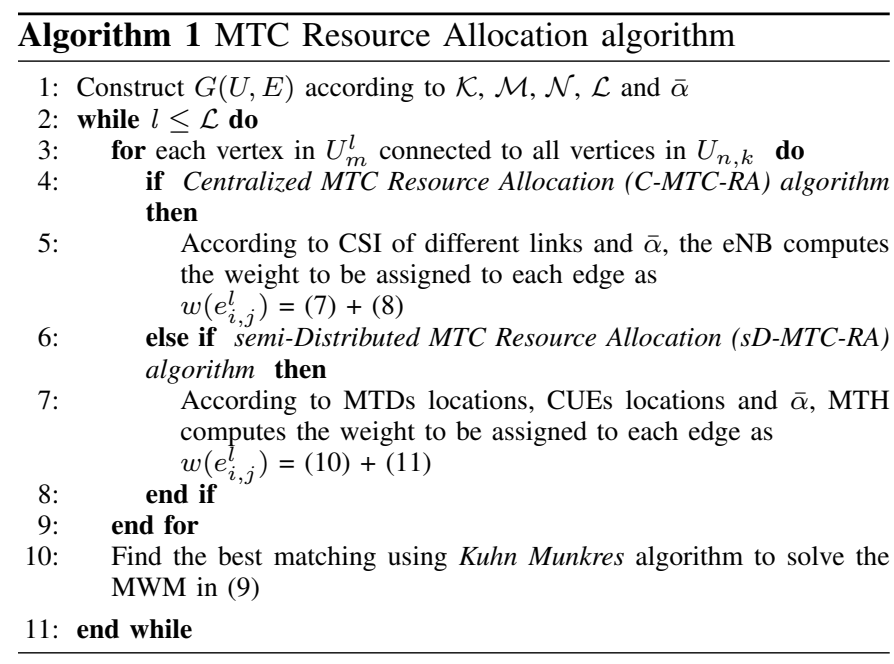

loss constant and a path-loss exponent, respectively. In this approach, each MTH executes in parallel and in a distributive way the Kuhn Munkres algorithm to find the corresponding optimal matching obtained in (9) through every scheduling process (see Algorithm 1).

\section{Complexity analysis}

The computational complexity of the proposed two-stage resource allocation approach is the sum of the complexity of both algorithms performed in each stage. In the first phase, the complexity of the $\mathrm{H} 2 \mathrm{H}$ resource allocation depends on the algorithm used. For instance, the PF algorithm allocates each RB after performing a linear search on the users in order to maximize a given utility function. Hence, the total complexity of the PF algorithm is $\mathcal{O}\left(N^{2} K\right)$. In the second stage, the computational complexity of the Kuhn Munkres algorithm applied for each MTH to solve the resource sharing problem is $\mathcal{O}\left(L N^{3}\right)$ which is polynomial. As a consequence, the proposed two-stage resource allocation scheme achieves lower computational costs comparing to the optimal method formulated in Section III-B.

\section{SIMULATION RESULTS AND ANALYSIS}

In order to evaluate the performance of both proposed algorithms, we consider an isolated cell, where $\mathrm{H} 2 \mathrm{H}$ communication and MTC within D2D communication coexist and share the RBs for their data transmission. The CUEs are distributed randomly within the network. MTDs are grouped into clusters, where there is one MTH per cluster. For demonstration purpose, the total number of MTDs per group is assumed to be the same. The detailed simulation parameters are given in table II. We consider the LTE-A bandwidth set to $20 \mathrm{MHz}$. The network performance in terms of sum-rate is evaluated in a scheduling period of 10 TTI. Thus, there is a total of $100 \mathrm{RBs}$ to be shared between MTC and H2H communication links. The simulation results are obtained through averaging over 100 different realizations of CUEs and MTDs location as well as channel gains using MATLAB environment. For simplicity, we suppose that both proposed MTC resource allocation algorithms are executed under the same conditions. Thus, the round robin $(R R)$ resource allocation algorithm is used for CUEs in the first phase, where all CUEs are served during a scheduling period.

\section{TABLE II: SIMULATION PARAMETERS}

\begin{tabular}{|c|c|}
\hline Parameter & Value \\
\hline \hline Cellular layout & Isolated cell \\
\hline Cell radius & $250 \mathrm{~m}$ \\
\hline Mobility & Static scenario \\
\hline Cluster radius & $\mathrm{N}=100 \mathrm{~m}$ \\
\hline CUEs per cell & $\mathrm{M}=70$ \\
\hline MTDs per cluster & UMi in [18] \\
\hline Path loss model & $24 \mathrm{dbm}$ \\
\hline UE transmit power $P_{U_{n}}$ & $15 \mathrm{dbm}$ \\
\hline MTD transmit power $P_{D_{m, t}}$ & $-174 \mathrm{dbm} / \mathrm{Hz}$ \\
\hline Noise power spectrum density & $2.5 \mathrm{Ghz}$ \\
\hline Carrier frequency & Rayleigh fading coefficient \\
\hline Small scale fading & with zero mean and unit variance \\
\hline Channel bandwidth & W=20 Mhz \\
\hline
\end{tabular}

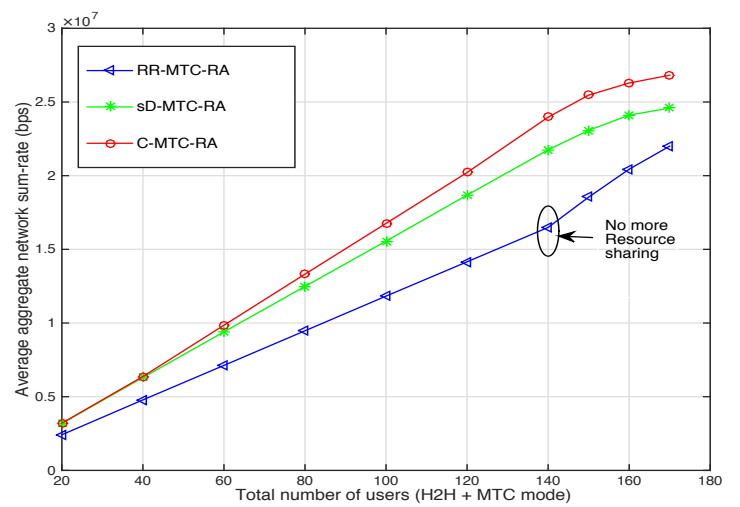

Fig. 3: Average network sum-rate (MTC $+\mathrm{H} 2 \mathrm{H}$ mode)

Fig 3 shows the average network sum-rate versus the total number of users when using only one cluster of MTDs. We compare the two proposed, centralized and semi-distributed, MTC based interference-aware BG resource allocation algorithms to the RR-MTC resource allocation algorithm (RRMTC-RA). In RR-MTC-RA, MTDs are scheduled according to a fixed pattern by scheduling MTDs one after another without taking into account the channel quality information. We see that the worst average network sum-rate is obtained for the RR-MTC-RA sharing algorithm. Indeed, RBs are allocated uniformly without considering any interference management mechanism. We also notice that the slope of the average network sum-rate for RR-MTC-RA algorithm slightly increases above 140 users since there is no more resource sharing ( $M$ MTDs have been served). We observe that the sD-MTC-RA algorithm can achieve almost similar performance compared to the C-MTC-RA algorithm. However, the proposed sD-MTCRA algorithm has lower communication overhead.

Fig 4 illustrates the average $\mathrm{H} 2 \mathrm{H}$ sum-rate versus the number of CUEs when introducing one cluster of MTDs. We 


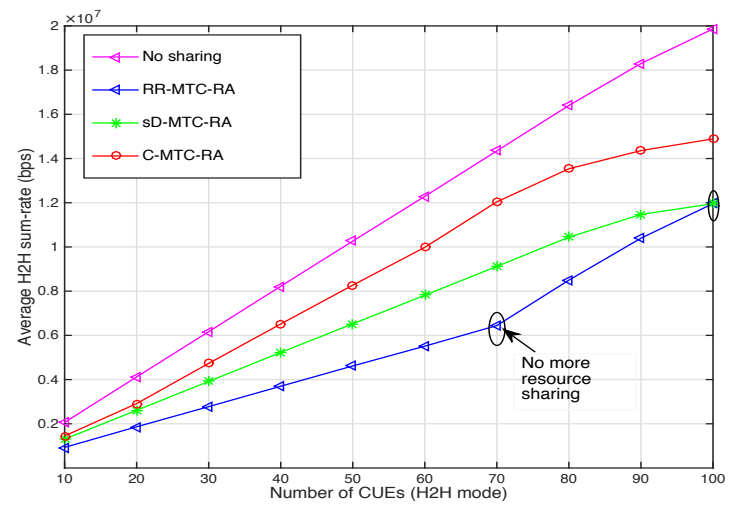

Fig. 4: Impact of MTC on average $\mathrm{H} 2 \mathrm{H}$ sum-rate ( $\mathrm{H} 2 \mathrm{H}$ mode)

aim to focus on the impact of introducing MTC on the $\mathrm{H} 2 \mathrm{H}$ performance when sharing the spectrum. As expected, we see that the worst performance is obtained when using the RRMTC-RA algorithm. For instance, below 70 users $(M=70)$, the $\mathrm{H} 2 \mathrm{H}$ sum-rate dramatically decreases. If we observe the evolution of the average $\mathrm{H} 2 \mathrm{H}$ sum-rate between the sD-MTCRA and the RR-MTC-RA algorithms, we observe that they coincide when all the RBs are used. This can be explained because the sum of the interference caused by MTC to $\mathrm{H} 2 \mathrm{H}$ when all RBs are used is almost similar. Indeed, both methods do not rely on the condition of the links, even though the sD-MTC-RA has shown better performance. In addition, we clearly see that the C-MTC-RA algorithm achieves better performance than the sD-MTC-RA algorithm since the C-MTC$\mathrm{RA}$ considers the accurate interference information on each $R B_{k}$. However, the C-MTC-RA has higher communication overhead than the sD-MTC-RA algorithm.

In order to justify why the inter-cluster interference has not been considered in the weight allocation process, we plot in Fig 5 the average MTC sum-rate for a maximum number of MTDs $(M=70)$ when only one cluster is used and the impact of introducing different clusters on the obtained average MTC sum-rate of one cluster. The positions of different clusters are generated randomly and the results are obtained through averaging over 100 realizations. Compared to the RR-MTCRA algorithm, both C-MTC-RA and sD-MTC-RA algorithms achieve better performance in terms of average MTC sumrate. The worst case is obtained when using the RR-MTCRA algorithm since RBs are assigned randomly to MTDs. As we expected, the higher is the number of clusters, the lower is the average MTC sum-rate. This result is quite reasonable since more MTDs are sharing the same spectrum. For instance, for a maximum number of $70 \mathrm{MTDs}$, the sum-rate difference is about $1 \mathrm{mbps}$ between the case where only one cluster is used $(M=70)$ and the case where 4 clusters $(L \times M=4 \times 70=280$ MTDs $)$ are emerged. Moreover, this result shows that the inter-cluster interference is negligible. On the other hand, we notice that contrarily to the average network sum-rate and the average $\mathrm{H} 2 \mathrm{H}$ sum-rate

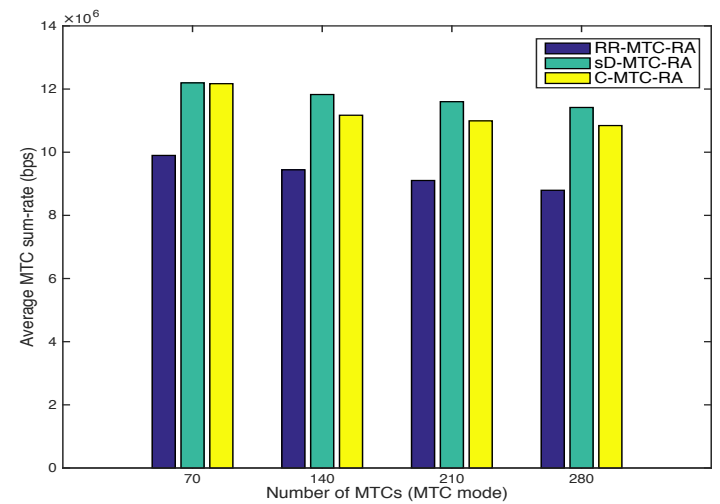

Fig. 5: Average MTD sum-rate when varying the number of clusters (MTC mode)

where the C-MTC-RA algorithm has been proved to achieve better performance compared to the sD-MTC-RA algorithm, this latter reaches slightly better performance in terms of MTC sum-rate compared to the C-MTC-RA algorithm which is quite surprising. However, by analyzing in detail what happens, this can be explained by the resource allocation process based on the mutual interference (interference from $\mathrm{H} 2 \mathrm{H}$ to $\mathrm{M} 2 \mathrm{M}$ and the interference from $\mathrm{M} 2 \mathrm{M}$ to $\mathrm{H} 2 \mathrm{H}$ ). Indeed, the interference introduced by $\mathrm{H} 2 \mathrm{H}$ communication is higher due to its high transmission power.

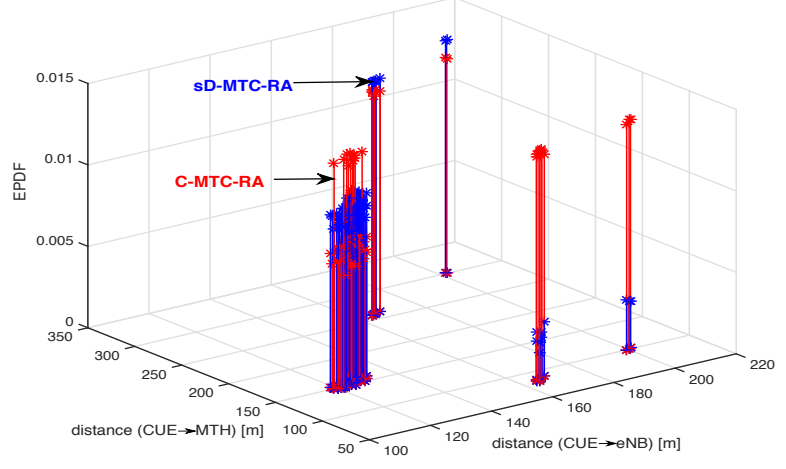

Fig. 6: Empirical probability density function (EPDF) of the distance of selected CUEs respectively to their eNB and MTH

Fig 6 represents the Empirical Probability Density Function (EPDF) of the distance of selected CUEs sharing the spectrum respectively to the $\mathrm{eNB}$ and $\mathrm{MTH}$. For the proposed sDMTC-RA algorithm, we observe that the density of CUEs sharing the spectrum increases the farther they are from the eNB and MTH. This result can be explained by the mutual interference weight allocation process that has been computed based on distances. However, we can notice that the density of CUEs sharing the spectrum in the proposed C-MTC-RA is fairly distributed. Indeed, the resource allocation process in the MTC-RA algorithm is based on the accurate channel quality 
of the links and not on distances.

\section{CONCLUSIONS}

In this work, we have proposed to combine the two emerging concepts enabled in LTE-A, MTC and D2D communication. We have investigated the resource sharing problem in an $\mathrm{M} 2 \mathrm{M} / \mathrm{H} 2 \mathrm{H}$ coexistence scenario. The computational complexity of the formulated optimal resource sharing problem has been settled to be NP hard. Hence, we have proposed a novel interference-aware BG based approach with polynomial computational complexity. To reduce the impact of MTC on traditional $\mathrm{H} 2 \mathrm{H}$ users, we have proposed a two-stage resource allocation approach. While conventional schedulers have been used for $\mathrm{H} 2 \mathrm{H}$ communication in the first stage, we developed two alternative MTC resource allocation algorithms in the second stage, one centralized and one semi-distributed. Simulation results have shown that the proposed semi-distributed MTC radio resource allocation algorithm achieves a good tradeoff between the network performance and the network overhead compared to the centralized MTC radio resource allocation algorithm, and also achieves significantly better performance compared to the random re-assignment approach. In addition, we have evaluated the inter-cluster interference and have shown that it is not significant. In the future work, we aim at analyzing our proposal under a vast set of scenarios with different mobility models.

\section{REFERENCES}

[1] M. Chen, "Towards smart city: $\mathrm{m} 2 \mathrm{~m}$ communications with software agent intelligence," Multimedia Tools and Applications, vol. 67, no. 1, pp. 167-178, 2013.

[2] M. Chen, J. Wan, and F. Li, "Machine-to-machine communications," KSII Transactions on Internet and Information Systems (TIIS), vol. 6 , no. 2, pp. 480-497, 2012.

[3] 3gpp ts 22.368 v10.1.0, "service requirements for machine-type communications," 2012

[4] 3gpp ts 22.888 v11.0.0, "system improvement for machine-type communication," 2012.

[5] G. T. 36.888, "Sudy on provision of low-cost machine-type communications (mtc) user equipements (ues) based on lte," 2012.

[6] N. Abu-Ali, A. Taha, M. Salah, and H. Hassanein, "Uplink scheduling in lte and lte-advanced: Tutorial, survey and evaluation framework," 2013.

[7] A. Asadi, Q. Wang, and V. Mancuso, "A survey on device-to-device communication in cellular networks," 2014.

[8] M. Hasan, E. Hossain, and D. Niyato, "Random access for machineto-machine communication in lte-advanced networks: issues and approaches," Communications Magazine, IEEE, vol. 51, no. 6, pp. 86-93, 2013.

[9] S.-Y. Lien and K.-C. Chen, "Massive access management for qos guarantees in 3gpp machine-to-machine communications," Communications Letters, IEEE, vol. 15, no. 3, pp. 311-313, 2011.

[10] S.-Y. Lien, K.-C. Chen, and Y. Lin, "Toward ubiquitous massive accesses in 3gpp machine-to-machine communications," Communications Magazine, IEEE, vol. 49, no. 4, pp. 66-74, 2011.

[11] A. S. Lioumpas and A. Alexiou, "Uplink scheduling for machine-tomachine communications in lte-based cellular systems," in GLOBECOM Workshops. IEEE, 2011, pp. 353-357.

[12] S. Zhenqi, Y. Haifeng, C. Xuefen, and L. Hongxia, "Research on uplink scheduling algorithm of massive $\mathrm{m} 2 \mathrm{~m}$ and $\mathrm{h} 2 \mathrm{~h}$ services in lte," 2013.

[13] 3gpp tr 36.211, "Evolved universal terrestrial radio access (e-utra); physical channels and modulation," 2010.

[14] R. Zhang, X. Cheng, L. Yang, and B. Jiao, "Interference-aware graph based resource sharing for device-to-device communications underlaying cellular networks," in Wireless Communications and Networking Conference (WCNC), 2013 IEEE. IEEE, 2013, pp. 140-145.
[15] D. Plaisted, "Some polynomial and integer divisibility problems are nphard," SIAM Journal on Computing, vol. 7, no. 4, pp. 458-464, 1978.

[16] C. Gueguen, A. Rachedi, and M. Guizani, "Incentive scheduler algorithm for cooperation and coverage extension in wireless networks," Vehicular Technology, IEEE Transactions on, vol. 62, no. 2, pp. 797-808, 2013.

[17] H. W. Kuhn, "The hungarian method for the assignment problem," Naval research logistics quarterly, vol. 2, no. 1-2, pp. 83-97, 1955.

[18] M. Series, "Guidelines for evaluation of radio interface technologies for imt-advanced," Report ITU, pp. 2135-1, 2009. 